\title{
Predictors of Poor Prognosis in Patients with Malignant Ascites: A Prospective Study
}

\author{
Abraham. A. Ayantunde*, Simon. L. Parsons \\ Department of Surgery, Nottingham University Hospitals, Nottingham City Hospital Campus, Hucknall Road, Nottingham, NG5 1PB, \\ United Kingdom
}

\begin{abstract}
Malignant ascites is a manifestation of end stage events in a variety of cancers with significant morbidity and a poor prognosis.This study prospectively evaluated factors predicting poor prognosis in patients with malignant ascites. Clinico-pathologic data of patients with malignant ascites at our institution over a period of 18 months were prospectively analysed. Predictors of poor survival were evaluated and survival compared among different cancer groups. A total of 150 patients (96 females, 54 males) with median age of 63 (19-95) years were studied. The commonest cancer was ovarian. Liver metastases were significantly commoner in the gastrointestinal cancers $(\mathrm{P}=0.0001)$. Serial paracentesis was offered to 131 , chemotherapy in 89, diuretics in 35, intraperitoneal monoclonal antibody in 6 and 7 patients had drainage at surgery. The median survival after the diagnosis of malignant ascites was 9.2 months. Ovarian cancer and female gender favour longer survival while poor performance status, low serum albumin/serum protein, liver metastases, high serum urea, creatinine and total bilirubin levels adversely affected survival. The independent prognostic factors for survival were poor performance status, cancer type, liver metastases, low serum albumin and high urea levels. The cancer type and performance status significantly influence the choice of treatment option.
\end{abstract}

Keywords Malignant Ascites, Cancers, Performance Status, Paracentesis, Chemotherapy, Poor Prognosis, Survival

\section{Introduction}

Malignant ascites signals end stage events in a wide spectrum of cancers and is associated with significant morbidity. It accounts for about $10 \%$ of all cases of ascites and usually caused by ovarian, breast, oesophageal, gastric, colorectal, lung, pancreatic, hepatobiliary, primary peritoneal, genitourinary carcinomas and malignancy of unknown primary[1-4]. We recently demonstrated in a retrospective study that more than $50 \%$ of patients with malignant ascites presented with ascites at the initial diagnosis of their cancer [4]. The onset and progression of malignant ascites is associated with deterioration in quality of life (QoL) and a poor prognosis. Few studies have reported on the natural history of malignant ascites but there are no clinical predictors that identify cancer patients who will develop this distressing entity; hence there are no preventive measures for its development.

There are different approaches to the treatment of malignant ascites but most are for symptomatic relief with few treating the underlying cancer. There is no generally accepted evidence based guidelines for evaluation and treatment of this condition. The main stays of treatment include

\footnotetext{
* Corresponding author:

biodunayantunde@yahoo.co.uk (Abraham. A. Ayantunde)

Published online at http://journal.sapub.org/cmd

Copyright (C) 2012 Scientific \& Academic Publishing. All Rights Reserved
}

symptomatic paracentesis, diuretics, systemic and intraperitoneal chemotherapy and peritoneovenous shunting. Recently, there are new emerging treatment concepts based on the increasing and better understanding of the aetiopathogenesis and pathophysiology of malignant ascites. It is envisaged that QoL and possibly the survival of patients with malignant ascites may be improved with increasing availability and use of appropriate multimodality therapy including potent combination chemotherapy. Only few studies have reported some factors affecting survival in patients with malignant ascites and are largely retrospective[4-8]. There is therefore need for a prospective study to evaluate the predictors of poor prognosis and survival in patients with malignant ascites.

\section{Objectives}

The objectives of this study were to determine the pattern of cancers causing malignant ascites and prospectively evaluate factors predicting poor prognosis.

\section{Methods}

Consecutive patients with malignant ascites treated at the Nottingham City Hospital over a period of 18 months were prospectively reviewed. The patients included in this study had clear confirmation of malignant origin of their ascites. 
Malignant origin of the ascites in these patients was confirmed by one or more of cytological examination, imaging, laparoscopy or laparotomy.

Clinico-pathological data of the patients were prospectively collected and predictors of poor survival were evaluated and survival compared among different cancer groups. The data collected included demographics, primary cancer entity, time interval between the diagnosis of the primary cancer and that of the ascites, methods of diagnosis of ascites, laboratory parameters at diagnosis, the presence of metastases at diagnosis of ascites, performance status as measured by Karnofsky index scoring system[9] and Eastern Cooperative Oncology Group (ECOG) performance scale[10], treatment of ascites and overall survival.

Statistical analysis was by the SPSS version 13.0 (SPSS, Chicago, Illinois, USA) using Mann-Whitney U-test and log rank test.Univariate and multivariate survival analyses were performed using the Kaplan-Meier survival function and Cox proportional hazard model with factors achieving p-value $<0.05$ considered significant.

\section{Results}

There were 150 patients with malignant ascites during this period with 96 (64\%) females and 54 (36\%) males. The median age at diagnosis of the ascites was 63 (19-95) years. The cancer types causing the ascites are shown in figure 1.

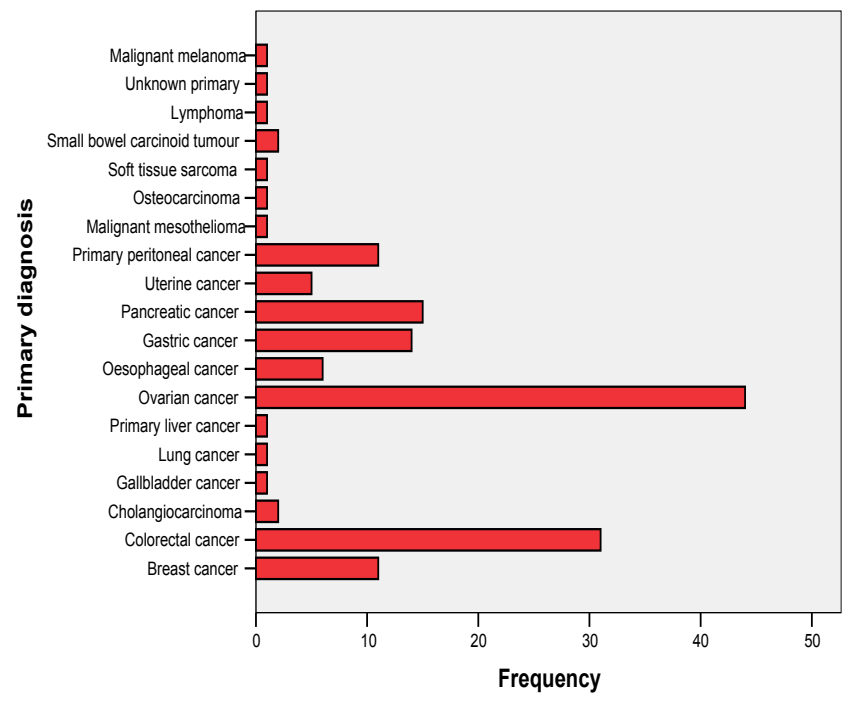

Figure 1. Primary cancer types causing malignant ascites

The median time interval between the diagnosis of the cancer of origin and that of the ascites was 1 (0-146) months. In fact $54 \%$ of patients had their ascites at first diagnosis of their cancer and the median of 1 (not 0 ) months represents a delay in diagnostic investigations in a few patients. Sixtyseven percent of the patients had their ascites diagnosed clinically and those with subclinical ascites, diagnosis was made at laparoscopy, laparotomy or with imaging investigations [Computerised tomography (CT) and ultrasound scans].
The sites of metastases at diagnosis of the ascites as demonstrated by diagnostic imaging techniques included peritoneal (126 patients), liver (46 patients), bone (10 patients), lung (16 patients), lymph nodes (11 patients). Thirty-one per cent of the patients had liver metastases and was commonest in patients with cancers of gastrointestinal origin at $78.3 \%$, followed by breast and ovarian cancers at $8.7 \%$ respectively and $4.3 \%$ in 'other' cancers. The difference in the incidence of liver metastases in the tumour groups is statistically significant $(\mathrm{P}=0.0001)$. Bone metastases were most common in patients with a breast primary. Immunocytochemistry of the ascites was positive in 99 $(66 \%)$, negative for cancer cells in $27(18 \%)$, suspicious in $13(8.7 \%)$ and was not performed in $11(7.3 \%)$. There was no metastasis detected in 9 patients on imaging techniques. Of these cytology was positive in 5 , negative in 2 and suspicious in 2 patients. The median total serum protein and albumin at diagnosis of the ascites in the 150 patients were 63 (41-80) $\mathrm{g} / 1$ and 26 (13-48) g/l respectively. The median serum urea, creatinine and total bilirubin at diagnosis of ascites were 5.7 $(2.2-33.8) \mathrm{mg} / \mathrm{dl}, 81(43-324) \mathrm{mg} / \mathrm{dl}$ and $(2-500) \mathrm{mg} / \mathrm{dl} \mathrm{re}-$ spectively. There is a significant correlation between the presence of liver metastases and low total serum protein $(p=0.026)$, low serum albumin $(p=0.005)$ and high total serum bilirubin $(\mathrm{p}=0.0001)$.

The most common treatment offered for the ascites was serial paracentesis in $131(87.3 \%)$ patients with mean of 2 (1-13) drainages required. Seven out of 150 (4.7\%) patients had the initial drainage of their ascites either at laparoscopy or laparotomy with only 1 requiring further paracentesis and the remaining 6 patients had no need for a further drainage procedure. These patients who had drainage at surgery or required no drainage at all during their hospital episode had subclinical ascites that were either diagnosed radiologically or incidentally found at laparoscopy or laparotomy. None of the patients in this series had either continuous catheter drainage or peritoneovenous shunt placement. Diuretics were used in 35 patients with spironolactone, an aldosterone antagonist used in $30(85.7 \%)$, frusemide in $2(5.7 \%)$ and combination of both in $3(8.6 \%)$. Diuretics tended to be used in patients with liver metastasis (33/46). Intravenous or oral single or combination chemotherapy was given in $89(59.3 \%)$ patients (37 ovarian, 26 gastrointestinal, 8 breast, 18 'other' cancers). The commonest chemotherapy in ovarian cancer was a carboplatin-based regimen. The remaining $40.7 \%$ of the patients were entered into the palliative care pathway and had only symptomatic control of their malignant disease process.

Follow up was until death or at least 7 months after diagnosis of ascites. There were 43 patients who were still alive at the end of the study (follow up range 7-61 months). The overall median survival after the diagnosis of malignant ascites was 7.17 (95\% CI, 4.53-9.81) months. Forty-nine out of 150 patients had subclinical ascites mostly from ovarian cancer and these had a significantly longer median survival than patients with clinical ascites $(\mathrm{p}=0.0001)$. Patients with 
ovarian cancer had better survival than those with other cancer groups $(\mathrm{P}=0.0001)$. Patients with ascites of gastrointestinal cancer origin generally had the worst survival (Figure 2).

Levels of serum albumin, total protein, urea, creatinine and bilirubin at diagnosis of ascites significantly affected survival. Low serum albumin (below $30 \mathrm{~g} / \mathrm{l}$ ) and low serum total protein (below $60 \mathrm{~g} / \mathrm{l}$ ) are both associated with poor overall survival $(\mathrm{P}=0.0001, \mathrm{P}=0.025$ respectively) while high levels of serum urea (above $6.5 \mathrm{mg} / \mathrm{dl}$ ), creatinine (above $100 \mathrm{mg} / \mathrm{dl}$ ) and bilirubin (above $17 \mathrm{mg} / \mathrm{dl}$ ) adversely affected long-term survival $(\mathrm{P}=0.0001, \mathrm{P}=0.001, \mathrm{P}=0.0001)$ respectively. The presence of liver metastases was associated with worse survival in all cancer groups $(\mathrm{P}=0.0001)$. Females survived longer than the males $(\mathrm{P}=0.0001)$ but this is likely to be due to better prognosis with ovarian and breast cancer groups. Patients with poor performance status either as classified by Karnofsky index score below 60 or ECOG score above 2 did worse $(\mathrm{P}=0.0001)$. There was improved survival in those patients who had chemotherapy $(\mathrm{P}=0.0001)$ but this again may be due to selection bias. Multivariate Cox regression analysis showed cancer type, low serum albumin levels, the presence of liver metastasis, patient performance status and high serum urea levels to be independent prognostic factors for overall survival [tables 1 and 2].

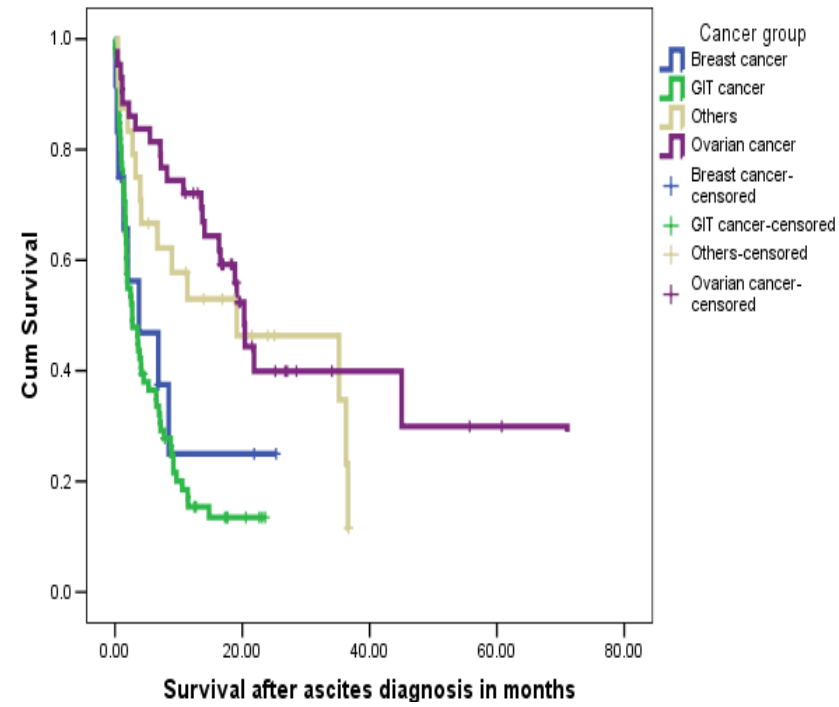

Figure 2. Kaplan-Meier survival curve comparing survival in patients with malignant ascites from different cancer groups

Table 1. Predictors of poor prognosis in patients with malignant ascites on univariate analysis

\begin{tabular}{|c|c|c|c|c|}
\hline \multicolumn{2}{|c|}{ Factors } & Alive & Dead & P-value \\
\hline \multirow{2}{*}{ Gender } & Female & 38 & 58 & \multirow{2}{*}{0.0001} \\
\hline & Male & 5 & 49 & \\
\hline \multirow{2}{*}{ Age } & $\leq 65$ years & 27 & 62 & \multirow{2}{*}{0.074} \\
\hline & $>65$ years & 16 & 45 & \\
\hline \multirow{4}{*}{ Cancer group: } & Breast cancer & 4 & 8 & \multirow{4}{*}{0.0001} \\
\hline & GIT cancer & 11 & 60 & \\
\hline & Ovarian cancer & 19 & 24 & \\
\hline & 'Other' cancer & 9 & 15 & \\
\hline \multirow{2}{*}{ Karnofsky index score: } & $\geq 60$ & 42 & 58 & \multirow{2}{*}{0.0001} \\
\hline & $<60$ & 1 & 9 & \\
\hline \multirow{2}{*}{ ECOG score: } & $1 / 2$ & 42 & 56 & \multirow{2}{*}{0.0001} \\
\hline & $3 / 4$ & 1 & 49 & \\
\hline \multirow{2}{*}{ Subclinical ascites: } & Yes & 31 & 18 & \multirow{2}{*}{0.0001} \\
\hline & No & 12 & 89 & \\
\hline \multirow{2}{*}{ Liver metastasis } & Yes & 5 & 41 & \multirow{2}{*}{0.0001} \\
\hline & No & 38 & 66 & \\
\hline \multirow{2}{*}{ Serum albumin levels } & Normal & 23 & 31 & \multirow{2}{*}{0.0001} \\
\hline & Low & 20 & 76 & \\
\hline \multirow{2}{*}{ Serum protein levels } & Normal & 28 & 48 & \multirow{2}{*}{0.0001} \\
\hline & Low & 15 & 59 & \\
\hline \multirow{2}{*}{ Serum urea levels } & Normal & 35 & 55 & \multirow{2}{*}{0.0001} \\
\hline & Low & 8 & 52 & \\
\hline \multirow{2}{*}{ Serum creatinine levels: } & Normal & 41 & 84 & \multirow{2}{*}{0.0001} \\
\hline & High & 2 & 23 & \\
\hline \multirow{2}{*}{ Serum bilirubin levels } & Normal & 40 & 83 & \multirow{2}{*}{0.0001} \\
\hline & High & 3 & 24 & \\
\hline
\end{tabular}

Table 2. Independent prognostic factors in patients with malignant ascites

\begin{tabular}{|c|c|c|c|c|}
\hline Factors & SE & P-value & RR & 95\% CI \\
\hline Primary cancer type & 0.143 & 0.017 & 1.711 & $1.026-2.941$ \\
\hline Performance status & 0.266 & 0.0001 & 4.537 & $2.694-7.639$ \\
\hline Liver metastasis & 0.261 & 0.034 & 1.620 & $1.372-3.035$ \\
\hline Serum albumin levels & 0.275 & 0.027 & 1.834 & $1.011-3.140$ \\
\hline Serum urea levels & 0.240 & 0.003 & 2.041 & $1.276-3.266$ \\
\hline
\end{tabular}




\section{Discussion}

Malignant ascites is a significant clinical problem with increasing burden in the oncological and palliative care medicine. It is associated with a progressively deteriorating quality of life (QoL) and a poor prognosis. However, with better understanding of the pathophysiology of malignant ascites, better diagnostic evaluation and the use of multimodality therapy, it is anticipated that the QoL and survival of these patients may be improved. There are few studies evaluating the natural history of malignant ascites and the prognostic factors relating to survival but they are largely retrospective[4-8].

We recently performed a retrospective study which showed that ovarian cancer was the commonest cause of ascites with far better prognosis than patients with gastrointestinal cancers. The study also demonstrated some other important prognostic factors affecting long term survival[4]. However, the study being retrospective was limited by not been able to evaluate the role of performance status and other biochemical parameters on the overall survival of patients with malignant ascites. The aim of the current study was therefore to evaluate prospectively prognostic factors affecting long term survival in patients with malignant ascites.

Again, the commonest cancer of origin leading to malignant ascites in the current series was ovarian representing $29 \%$ of the total patient population. There is a predominance of the female patient population at $64 \%(96 / 150)$ similar to our previous studies[4,5] and this is accounted for by the predominance of the ovarian and breast cancers in the study population. Fifty-four per cent of our patient population presented with malignant ascites at the initial diagnosis of their cancer and this is similar to the series previously reported[4,5]. These patients were mainly the ovarian and the gastrointestinal cancer groups, while patients with breast cancer tended to develop ascites due to their cancers months or years after their primary cancer had been diagnosed and treated. There was only one patient with malignant ascites due to an unknown primary cancer in the present study and this is far less than the proportions in the previous studies reported at $8-23 \%[4,5,11]$. This is probably due to improvements in determination of tumour of origin by multimodal techniques such as imaging, laparoscopy and immunocytochemical analysis. In this prospective series, 9 patients had no evidence of metastases on imaging. However 7 out of the 9 had malignant or suspicious cytology and therefore, probably had peritoneal carcinomatosis not detected on CT. Cytology was negative in two patients but they had clinical and imaging evidence of the primary tumour with no other cause of their ascites. It may be that the cytologically negative patients also had peritoneal carcinomatosis that was not picked up by imaging investigations, although Runyon et al[3] previously showed that of their total number of patients with malignant ascites, 53.3\% had peritoneal carcinomatosis and all of this group had a positive cytology suggesting a near $100 \%$ sensitivity of cytology in patients with peritoneal carcinomatosis.

Sixty-eight $(45 \%)$ of our patients are still alive after a minimum follow up of 7 months and most of them are in the ovarian cancer group. Ovarian cancer is usually amenable to debulking surgery and has a good response to chemotherapy. Our finding that ascites of ovarian origin has a better median survival than all other cancer groups agrees with previous studies[4-8]. This also may have been responsible for the better survival seen in women compared to men. $86 \%(37 / 43)$ of our patients with ovarian cancer had chemotherapy which was mainly a carboplatin based regime with a measure of response.

We have prospectively evaluated various factors that affect survival in patients with malignant ascites and our findings apart from factors identified by previous studies [4-8] has shown the significant role of performance status and other biochemical parameters. Patients with gastrointestinal cancer particularly have a very poor survival compared with the ovarian cancer group. There is an increasing use of aggressive multimodality therapy including combination chemotherapy, 59\% received chemotherapy in the current study compared to $49 \%$ and $41 \%$ in our previous studies respectively[4,5]. Furthermore, there is a progressive improvement in the success rates of the use of chemotherapy especially in breast and ovarian cancers. Patients who had chemotherapy also survived longer than those who did not receive chemotherapy but again this is likely to be multifactorial such as response of cancer and ascites to chemotherapy, the higher proportion of the ovarian cancer in the chemotherapy group and the fact that patients selected for chemotherapy are those with relatively good performance status and longer life expectancy.

The presence of liver metastases at the time of ascites diagnosis was a significant predictor of poor survival both on univariate and multivariate analyses. This finding was similar to that from previous studies[4-6,8]. Patients with gastrointestinal cancer were found to be more likely to have liver metastases and accounted for $78 \%$ of cases with hepatic metastases. Patients with liver metastases in this study tended to be given diuretics on the understanding that their ascites is likely caused by the renin-angiotensin-aldosterone pathway in some similar fashion to cirrhotic ascites. Diuretics have been shown to be more effective in the presence of liver metastases[2,12,13]. Low levels of serum albumin and total proteins are significant factors affecting survival adversely. In fact low serum albumin is an independent prognostic factor especially in the non-ovarian cancer groups. There are no other studies that have reported the prognostic roles of serum albumin and total protein in malignant ascites except our previous studies[4,5]. The poor prognostic role is likely to be related to poor nutritional reserve, association of low total serum protein with the presence of liver metastases and impaired immune network function in this group of patients[4]. Our findings in the current study and as previously shown also demonstrated that female gender is associated with longer survival and a better prognosis and we 
believe that this is due to the combined preponderance of ovarian and breast cancer groups.

Karnofsky performance index scale or ECOG performance score are two popular indices for clinical estimation of a patient's physical state, performance and prognosis $[9,10]$. They are useful tools for determining a patient's suitability for therapy and measuring outcome. Performance status either as evaluated by Karnofsky performance index score or Eastern Cooperative Oncology Group (ECOG) performance score has been hereby shown to be a significant predictor of survival in patients with malignant ascites. Patients with poor performance status generally had worse overall survival. Performance status score is an important tool in predicting patients' life expectancy, selection for multimodality treatment of malignant ascites and prognosis. Patients with high serum creatinine, urea and bilirubin, an indication of multiorgan dysfunction have worse overall survival. Patients' selection and the choice of treatment option for patients with malignant ascites will be greatly enhanced by applying these identified factors. The patients who had no chemotherapy $(41 \%)$ in this series generally are those with poor performance status and were entered into the palliative care pathway and had only symptomatic control of their symptoms from the malignant disease process.

Serial paracentesis was still the commonest treatment offered to patients with symptomatic malignant ascites followed by chemotherapy and the use of diuretics. Paracentesis provides relief of symptoms for patients with malignant ascites and improves their quality of life[14]. Lee et al[15] in a survey of Canadian physicians' practice showed that the use of diuretics was the second preferred treatment after therapeutic paracentesis though it was not felt to be effective in the treatment of malignant ascites. Current study clearly showed that chemotherapy is becoming popular as a dominant treatment options in carefully selected patients with malignant ascites and this is clearly due to progressive success rates in the use of new chemotherapeutic agents for cancers. The group of patients who had drainage at surgery and required no further drainage and those who required no drainage at all during the course of their hospital episode were those with subclinical ascites that were either diagnosed radiologically or incidentally found at laparoscopy or laparotomy. There is no prospective study evaluating the role of diuretics and no general consensus for the use of diuretics in the treatment of malignant ascites. Therefore further studies are required in order to identify those patients who will benefit from this therapy. Six patients in this study had intraperitoneal monoclonal antibody (Catumaxumab) against epithelial cell adhesion molecule (EpCAM) as part of a large multicentre randomised phase II/III clinical trial. The results of this clinical trial are eagerly awaited. None of the patients evaluated had intracavitary chemotherapy, radiotherapy or matrix metalloproteinases inhibitors (MMPs). The role of intraperitoneal chemotherapy for treating malignant ascites is increasingly being advocated. Intraperitoneal chemotherapy is more effective in a situation when the tumour has been previously responsive to systemic chemo- therapy especially in ovarian and breast cancer ascites[16]. A therapeutic effect is best achieved in the state of minimal or microscopic tumour load and setting of hyperthermia because of limited drug penetration[17]. The results from these agents used intraperitoneally are encouraging but there are no large series on this regimen of treatment for malignant ascites. There is therefore need for large multicentre randomized trials to further evaluate this therapy in malignant ascites in order to assess its efficacy and draw evident and meaningful conclusion.

\section{Conclusions}

The overall prognosis of patients with malignant ascites is poor and patients are typically in the palliative phase of care when they present. However, patients may be successfully palliated by careful selection and appropriate offer of therapy tailored towards individual patient's characteristics and primary cancer type. The use of identified predictors of poor prognosis will assist the clinician in patients' selection and the choice of treatment option. The prognosis in malignant ascites is significantly determined by the origin of the primary cancer, performance status, presence of hepatic metastases and serum albumin concentration. Malignant ascites of ovarian origin has a better prognosis while patients with malignant ascites of gastrointestinal origin have the worst outcome. The independent prognostic factors mentioned above should be used to select patients for multimodality therapy as this may offer adequate palliation, improved quality of life and prolonged survival.

\section{REFERENCES}

[1] Runyon, B.A; 1994, Care of patients with ascites. N Engl J Med; 330: 337-342.

[2] Parsons, S.L, Watson, S.A and Steele, R.J.C; 1996, Malignant ascites (Review). Br J Surg; 83: 6-14.

[3] Runyon, B.A, Hoefs, J.C and Morgan, T.R; 1988, Ascitic fluid analysis in malignancy-related ascites. Hepatology; 8: 1104-1109.

[4] Ayantunde, A.A and Parsons, S.L; 2007, Pattern and prognostic factors in patients with malignant ascites: a retrospective study. Annals of Oncology; 18(5) 945-949.

[5] Parsons, S.L, Lang, M.W and Steele, R.J.C; 1996, Malignant ascites: a 2 year review from a teaching hospital. Eur J Surg Oncol; 22:237-239.

[6] Mackey, J.R and Venner, P.M; 1996, Malignant ascites: demographics, therapeutic efficacy and predictors ofsurvival. Can J Oncol; 6: 474-480.

[7] Wilailak, S, Linasmita, V and Srivannaboon, S; 1999, Malignant ascites in female patients: a seven-year review. J Med Assoc Thai; 82: 15-19.

[8] Appelqvist, P, Silvo, J, Salmela, L and Kostiainen, S; 1982, 
On the treatment and prognosis of malignant ascites:is the survival time determined when the abdominal paracentesis is needed? J Surg Oncol; 20: 238-242.

[9] Karnofsky, D.A and Burchenal, J.H. The clinical evaluation of chemotherapeuticagents in cancer.In: MacLeod CM (Ed), 'Evaluation of Chemotherapeutic Agents'.Columbia Univ Press, 1949: 196.

[10] Oken, M.M, Creech, R.H, Tormey, D.C, Horton, J, Davis, T.E, McFadden, E.T and Carbone, P.P; 1982, Toxicity andresponse criteria of the Eastern CooperativeOncology Group (ECOG). Am J Clin Oncol; 5: 649-655.

[11] Ringenberg, Q.S, Doll, D.C, Loy, T.S, Yarbro, J.W; 1989, Malignant ascites of unknown origin. Cancer 1989; 64: 753-755.

[12] Greenway, B, Johnson, P.J and Williams, R; 1982, Control of malignant ascites with spironolactone. Br J Surg; 69: 441-442.
[13] Pockros, P.J, Esrason, K.T, Nguyen, C, Duque, J and Woods, S; 1992, Mobilization of malignant ascites withdiuretics is dependent on ascitic fluid characteristics.Gastroeneterology; 103: $1302-1306$.

[14] Easson, A, Bezjak, A, Ross A, Sam, F, Newman, A, Bana, N and Wright, J.G; 2005, Changes in symptoms afterparacentesis for symptomatic malignant ascites. J Clin Oncol; 16: 6071 (Suppl).

[15] Lee, C.W, Bociek, G and Fraught W; 1998, A survey of practice in management of malignant ascites. J Pain Symptom Management; 16: 96-101.

[16] Adam, R.A and Adam, Y.G; 2004, Malignant ascites: Past, Present and Future.J Am Coll Surg; 6: 999-1011.

[17] Garofalo, A, Valle, M, Garcia, J and Sugarbaker, P.H; 2006, Laparoscopic intraperitoneal hyperthermia chemotherapy for palliation of debilitating malignant ascites. EJSO; 32: 682-685. 